\title{
Identification of Elastic Parameters by Treating the Inverse Problem
}

\author{
El hanafi ARJDAL \\ Laboratory of Electronics, \\ Signal Processing and \\ Modelling Physics \\ Department of Physics \\ University Ibn Zohr \\ Agadir ,Morocco
}

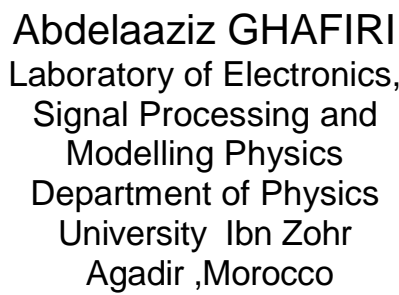

\author{
Jamal CHAOUFI \\ Laboratory of Electronics, \\ Signal Processing and \\ Modelling Physics \\ Department of Physics \\ University Ibn Zohr \\ Agadir ,Morocco
}

\author{
Arnaud GERMANEAU \\ Institut $P$ ' \\ University of Poitiers \\ Poitiers, France
}

\author{
Claude VALLEE \\ Institut $P^{\prime}$ \\ University of Poitiers \\ Poitiers, France
}

\author{
Hassane FATMAOUI \\ Laboratory of Electronics, \\ Signal Processing and \\ Modelling Physics \\ Department of Physics \\ University lbn Zohr \\ Agadir,Morocco
}

\begin{abstract}
The aim of this work is to lay the groundwork for identifying digital mechanical parameters of materials with elastic. Most of the tests do not allow identifying these parameters automatically. The use of the finite elements of calculations for sizing works is thus limited by a poor understanding of the mechanical properties. In this context, it raises the issue of inverse analysis [1] [2]. From this information about the parameters of the laws of material behavior, is it possible to obtain the displacement field from in situ measurements and how does digital technology obtain a determination of these parameters accurately and systematically?
\end{abstract}

In this work we present a new approach by providing a formulation is easily used by treating the inverse problem. It is based on the finite element method, which, in a direct problem, gives the displacement field knowing the mechanical properties and an inverse problem gives the mechanical knowledge of the field trips.

The resolution of the direct problem has yielded results. The latter is in agreement with the simulation code of commercial calculation. This allowed us to address the inverse problem with no understanding by offering an alternative identification using a database previously determined [3].

\section{Keywords}

Mechanical parameters, identification, direct problem, the inverse problem

\section{INTRODUCTION}

The mechanical properties of materials are the focused point because they determine not only the problems but also shaping their behavior during their service in extremely diverse industrial applications. The appropriate choice of the materials for an industrial part depends on the mechanical properties, strength, hardness, ductility ... It is therefore necessary to accurately measure these physical quantities by mechanical tests.
The determination of these properties to design and model the mechanical parts is necessary for efficiency, performance and design.

Indeed, in response to these powers we propose a new way of identification. It consists in using the finite elements of a method as a basis to develop a model that meets the above requirements. This writing will help us to simplify the boundary value problem for a differential equation problem resolved easily and can be used for the inverse problem. This approach can be extended to amore complex behavior materials such as composites.

\section{POSITION OF THE PROBLEM}

\subsection{Strong formulation}

Before addressing the problem by finite elements we are forced to go through the strong formulation that comes down to this.

Find the displacement field $\vec{U}$ in a field $\Omega$ border $\partial \Omega$ such as:

In $\Omega$ the balance equation $\operatorname{div} \bar{\sigma}+\vec{f}=\overrightarrow{0}$ connecting the stress tensor $\bar{\sigma}$ and volume forces $\vec{f}$.

The elastic constitutive law between the stress tensor $\overline{\bar{\sigma}}$ and the strain tensor $\stackrel{\bar{\varepsilon}}{\varepsilon}$ :

$\overline{\bar{\sigma}}=A \bar{\varepsilon}=\lambda \operatorname{tr}(\bar{\varepsilon}) \overline{\bar{\varepsilon}})=$

Where $\overline{\bar{I}}$ is the identity tensor, and $A$ the flexibility matrix. The boundary conditions are:

$\vec{U}=\vec{U}_{0}$ on $S_{u}$

$\bar{\sigma} \vec{n}=\vec{p}$ on $S_{\sigma}$

As $\partial \Omega=S_{u} \cup S_{\sigma}$ and $S_{u} \cap S_{\sigma}=\varnothing$

\subsection{Matrix formulation}

Using the convention Voigt matrix vector displacements, strains and stresses are written respectively in a twodimensional pattern by: 
$u=\left(\begin{array}{l}u_{x} \\ u_{y}\end{array}\right), \varepsilon=\left(\begin{array}{c}\varepsilon_{x} \\ \varepsilon_{y} \\ 2 \varepsilon_{x y}\end{array}\right), \sigma=\left(\begin{array}{c}\sigma_{x} \\ \sigma_{y} \\ \sigma_{x y}\end{array}\right)$

To make the flexibility matrix diagonal, we adopt a new convention, whose base is formed by two spherical and deviatoric. The base is chosen is the following:

$E_{1}=\frac{1}{\sqrt{2}}\left[\begin{array}{ll}0 & 1 \\ 1 & 0\end{array}\right], E_{2}=\frac{1}{\sqrt{2}}\left[\begin{array}{cc}1 & 0 \\ 0 & -1\end{array}\right]$ et $E_{3}=\frac{1}{\sqrt{2}}\left[\begin{array}{ll}1 & 0 \\ 0 & 1\end{array}\right]$

Thus, the vectors of stress and strain that we denote respectively by $Y$ and $X$ be written into the new database:

$$
Y=\left(\begin{array}{c}
Y_{1}=\sqrt{2} \sigma_{x y} \\
Y_{2}=\frac{1}{\sqrt{2}}\left(\sigma_{x}-\sigma_{y}\right) \\
Y_{3}=\frac{1}{\sqrt{2}}\left(\sigma_{x}+\sigma_{y}\right)
\end{array}\right) \text { and } X=\left(\begin{array}{c}
X_{1}=\sqrt{2} \varepsilon_{x y} \\
X_{2}=\frac{\varepsilon_{x}-\varepsilon_{y}}{\sqrt{2}} \\
X_{3}=\frac{\varepsilon_{x}+\varepsilon_{y}}{\sqrt{2}}
\end{array}\right)
$$

Within the base of the matrix diagonal and flexibility is of the form:

$$
A=\left[\begin{array}{ccc}
2 \mu & 0 & 0 \\
0 & 2 \mu & 0 \\
0 & 0 & 2(\lambda+\mu)
\end{array}\right]
$$

Where $\lambda$ and $\mu$ are the Lame coefficients.

\subsection{Weak formulation}

To address the problem by finite elements, the weak formulation is very useful. It is to rewrite the formulation as high energy using the principle of virtual work, which states:

The work of external forces $\delta \tau$ is equal to the deformation energy $\delta w: \delta w=\delta \tau$

where

$$
\delta \tau=\int_{v} f_{i} \delta u_{i} d v+\int_{s_{\sigma}} \delta u_{i} p_{i} d s=\int_{v} f_{i} \delta u_{i} d v+\int_{s_{\sigma}}\{\delta u\}^{t}\{p\} d s
$$

with $p_{i}=\sigma_{i j} n_{j}$

and

$$
\begin{gathered}
\left.\delta w=\int_{v} \delta \varepsilon_{i j} \sigma_{i j} d v=\int_{v}\{\delta \varepsilon\}^{t}\{\sigma\} d v=\int_{v}\{\delta u\}^{t}[\partial][A\rceil \partial\right]\{u\} d v \\
\text { with }[\partial]=\left(\begin{array}{cc}
\frac{\partial}{\partial Y} & \frac{\partial}{\partial X} \\
\frac{\partial}{\partial X} & -\frac{\partial}{\partial Y} \\
\frac{\partial}{\partial X} & \frac{\partial}{\partial Y}
\end{array}\right) \text { derivative operator is written in }
\end{gathered}
$$

matrix form in the basis $\left(E_{1}, E_{2}, E_{3}\right)$.

Determining the displacement field amounts to solving the linear system given by the finite element method; adopted elements are triangles with three nodes:

$[K]\{u\}=\{F\}$

such that:

$[K]=\sum_{e} K^{e}=\sum_{e}\left[P^{e}\right]^{t}\left[k^{e}\right]\left[P^{e}\right]$ is the global stiffness matrix,

$\left[P^{e}\right]$ is the matrix of the positioning member (e) in the overall structure.
$\left[k^{e}\right]=\int_{\Omega e}\left[B^{e}\right]^{t}[A]\left[B^{e}\right] d v \quad$ is the elementary stiffness matrix.

$\left[B^{e}\right]=[\partial]\left[N^{e}\right]$ is the gradient of the interpolation functions. $\left[N^{e}\right]$ is the matrix of elementary interpolation functions.

$\{u\}$ is the vector of nodal displacements in the overall structure.

$\{F\}=\sum_{e}\left[P^{e}\right]^{T}\left\{f^{e}\right\}$ is the vector of global nodal forces.

with $\left\{f^{e}\right\}=\int_{S \sigma}[N]^{T}\{p\} d s$.

Determining the scope of displacement amounts to solving the linear equation $[K]\{u\}=\{F\}$.

with boundary conditions:

$\vec{U}=\vec{U}_{0} \quad$ on $S_{u}$

$\overline{\bar{\sigma}} \cdot \vec{n}=\vec{p} \quad$ on $S_{\sigma}$

as $\partial \Omega=S_{u} \cup S_{\sigma}$ and $S_{u} \cap S_{\sigma}=\varnothing$

This equation is much more accessible to its simplicity.

\section{RESULTS}

Consider a homogeneous solid rectangular plate having a thickness e, the length $\mathrm{L}$ and width $\mathrm{h}$. Shifts in the distribution of the plate is measured in 2-D. plate occupies the interval $[0$, L] of the Ox axis, $[0, h]$ of the axis Oy demands are varied and must be independent. Below are three examples of types of load applied to the plate:
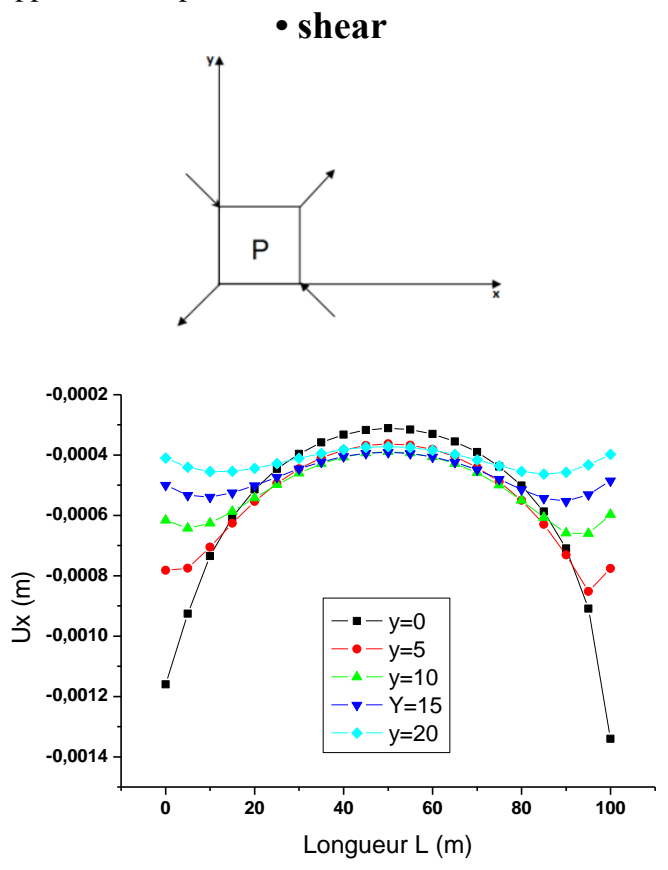

Fig. 1 Axial displacement function of $x$ 


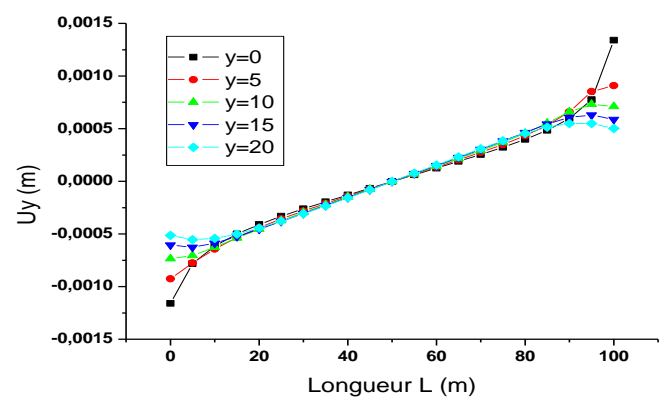

Fig. 2 Displacement along y axis

- Traction diagonal
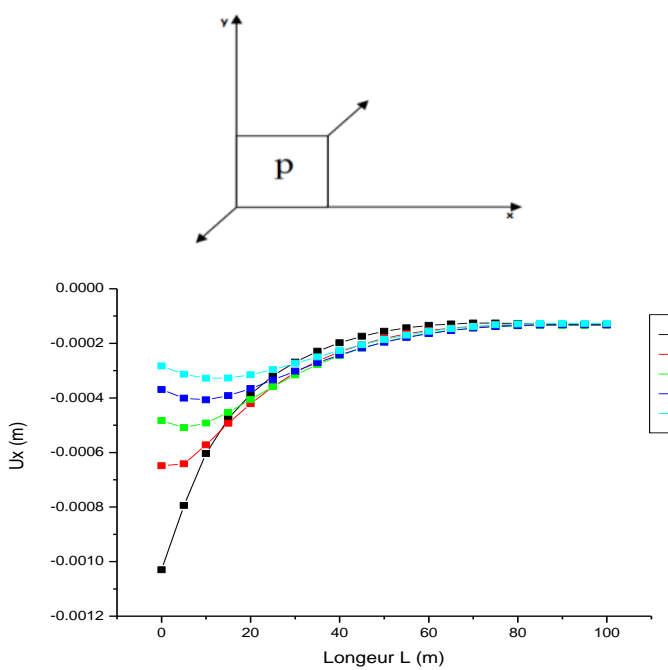

Fig. 3 Axial displacement function of $x$

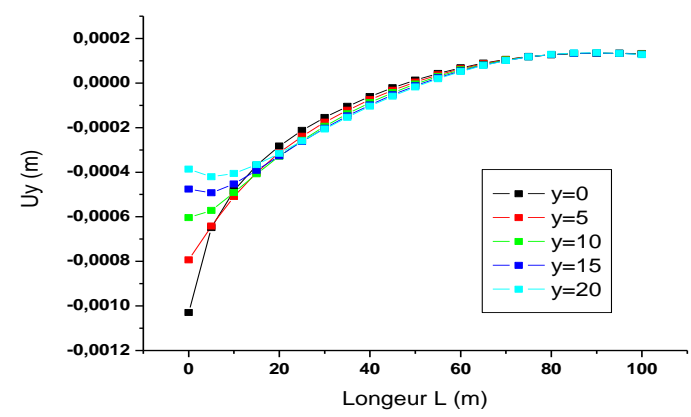

Fig. 4 Displacement along $y$ axis

- Traction biaxial

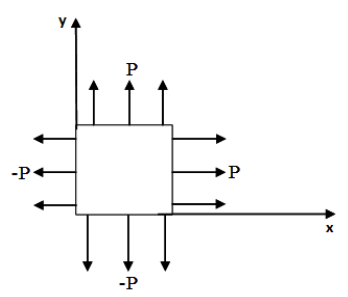

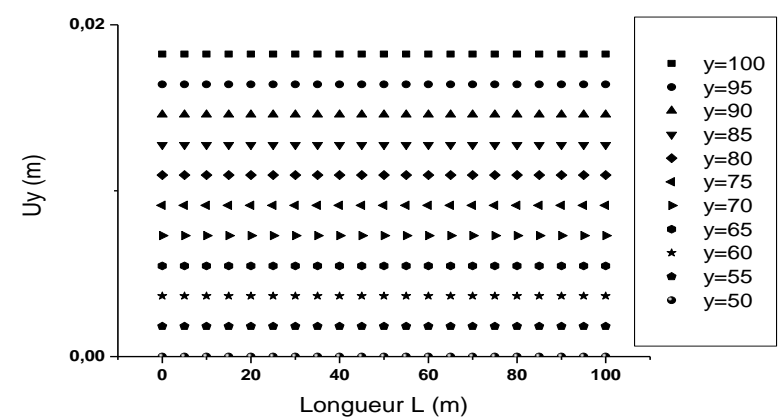

Fig 5. Displacement along y axis

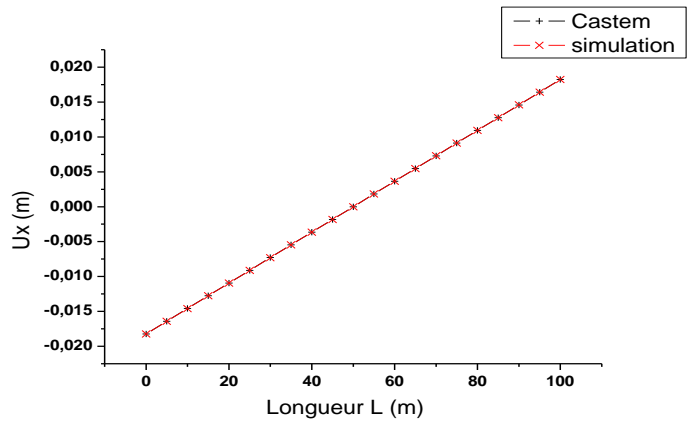

Fig 6. Axial Displacement function of $x$

The comparison with the results given by a code of commercial calculation (CAST3M) for example tensile biaxial validates our approach (Figure 6). This gives substantially the same displacement and that the three types of stress (Figures 1-5).

\section{FORMULATION OF THE INVERSE PROBLEM}

The goal of the inverse problem is to find the coefficients $\lambda$ and $\mu$ matrix $A$ in the behavior law.

Note that $A$ is diagonal and positive definite if $\mu>0$ and $\lambda+\mu>0$. First, we seek to identify $[K]$,

the finite element method, knowing the nodal displacements $\{u\}$ and nodal forces $\{F\}$ solutions of the linear system $[K]\{u\}=\{F\}$. Then find $A$ by minimizing the function $\frac{1}{2}\left\|K-\sum_{e} \int_{\Omega e}\left[P^{e}\right]^{T}\left[B^{e}\right]^{T}[A]\left[B^{e}\right]\left[P^{e}\right]\right\|^{2}$

Suppose have i experience as $[K]\left\{u_{i}\right\}=\left\{F_{i}\right\}$; if we multiply this by right relationship $\left\{u_{i}\right\}^{t}$ and if the sum of all the experiences i we obtain the following system:

$$
[K] \underbrace{\sum_{i}\left\{u_{i}\right\}\left\{u_{i}\right\}^{t}}_{H}=\underbrace{\sum\left\{F_{i}\right\}\left\{u_{i}\right\}^{t}}_{B}
$$

to determine $[K]$ must be chosen so that sufficient experience $[H]$ is invertible in these conditions 


$$
[K]=[B \perp H]^{-1} \quad \text { where } \quad[H]=\sum_{i}\left\{u_{i}\right\}\left\{u_{i}\right\}^{t} \text { and }
$$$$
[B]=\sum\left\{F_{i}\right\}\left\{u_{i}\right\}^{t}
$$

The identification of the elasticity tensor $\mathrm{A}$ is equivalent to solving the following linear system:

$$
\sum_{e} \int_{\Omega e}\left[P^{e}\right]^{T}\left[B^{e}\right]^{T}[A]\left[B^{e}\right]\left[P^{e}\right] d v=K \Leftrightarrow L(A)=K
$$

$\mathrm{L}$ is a linear operator.

$$
A=2 \mu \underbrace{\left(\begin{array}{lll}
1 & 0 & 0 \\
0 & 1 & 0 \\
0 & 0 & 1
\end{array}\right)}_{A_{1}}+2 \lambda \underbrace{\left(\begin{array}{lll}
0 & 0 & 0 \\
0 & 0 & 0 \\
0 & 0 & 1
\end{array}\right)}_{A_{2}}
$$$$
[A]=\sum_{i=1}^{2} h_{i}\left[A_{i}\right] \text { with } h_{1}=2 \mu \text { and } h_{2}=2 \lambda
$$

As

$$
[K]=\sum_{i=1}^{2} h_{i} \sum_{e} \int_{\Omega e}\left[P^{e}\right]^{T}\left[B^{e}\right]^{T}\left[A_{i}\right]\left[B^{e}\right]\left[P^{e}\right] d v=\sum_{i=1}^{2} h_{i} H_{i}
$$

Our process is based identification sue the method of least squares. It is to determine the matrix $A$, minimizing the quadratic form:

$J(h)=\frac{1}{2}\left\|\sum_{i=1}^{2} h_{i} H_{i}-K\right\|^{2}$ called cost function.

It is written below in more detail:

$$
J(h)=\frac{1}{2}\left\|\sum_{i=1}^{2} h_{i} H_{i}-K\right\|^{2}=\frac{1}{2} h^{T} S h-h^{T} V+\frac{1}{2} \operatorname{tr}\left(K K^{T}\right)
$$

where the components of the matrix $S$ and the vector $V$ are respectively $S_{i j}=\operatorname{tr}\left(H_{i} H_{j}^{T}\right)$ and $V_{i}=\operatorname{tr}\left(H_{i} K\right)$

We note that, $K, H_{1}$ et $H_{2}$ are symmetric and $\mathrm{S}$ is symmetric positive. Thus the cost function $J(h)$ convexe. is its derivative is written as:

$\operatorname{grad} J(h)=S h-V$

One solution to this problem is to minimize the cost function: $J(h)=\frac{1}{2} h^{T} S h-h^{T} V+\frac{1}{2} \operatorname{tr}\left(K K^{T}\right)$

with taking into account inequalities: $h_{1} \geq 0, h_{2} \geq 0$

and if we set $g_{1}(h)=h_{1}, g_{2}(h)=h_{2}$

Minimize the problem of starting with constraints in the sense $\geq$ is as good situation to maximize with constraints in the direction $\leq$.In this case the multiplier is positive

$\overrightarrow{\operatorname{grad}} g_{1}(h)=e_{1}=\left(\begin{array}{l}1 \\ 0\end{array}\right), \overrightarrow{\operatorname{grad}} g_{2}(h)=e_{2}=\left(\begin{array}{l}0 \\ 1\end{array}\right)$

The formulation of the inverse problem amounts to solving the following problem by introducing the Kuhn-Tucker multiplier $\lambda_{1}$ and $\lambda_{2}$.

For a general solution must be found $\left(h_{1}, h_{2}, \lambda_{1}, \lambda_{2}\right)$ Solutions:

$$
\left\{\begin{array}{c}
\overrightarrow{\operatorname{grad} J}=\sum_{i=1}^{2} \lambda_{i} \overrightarrow{\operatorname{grad}} g_{i}(h)=\lambda_{1} e_{1}+\lambda_{2} e_{2}=\left(\begin{array}{l}
\lambda_{1} \\
\lambda_{2}
\end{array}\right)=S h-V \\
\lambda_{1} h_{1}=0 \\
\lambda_{2} h_{2}=0 \\
\lambda_{1} \geq 0, \lambda_{2} \geq 0, h_{1} \geq 0, h_{2} \geq 0
\end{array}\right.
$$

\section{CONCLUSION}

This work has allowed us to validate our approach based on the finite element method and build a database used by the inverse problem. The identification and modeling systems from experimental data is an active area of research in several areas, especially in materials. The aim of the identification is then to provide an estimate of the mathematical model of the system considered in order to simulate, to order or to determine in a systematic way the physical parameters of a material. The finite element method is essential to this kind of problem. The direct formulation of the problem is completed giving satisfactory results. It nevertheless remains to be validated in real cases. The inverse problem is posed and is also completely to apply to concrete cases.

\section{REFERENCES}

[1] M. Grediac, F. Pierron Applying the Virtual Fields Method to the identification of elasto-plastic constitutive parameters. International Journal of Plasticity. Volume: 22. Pages: 602-627. 2006.Elsevier.

[2] S. Avril , M.Bonnet, A.-S Bretelle., M.Grediac, F.Hild, P.Ienny, F.Latourte, D.Lemosse, E.Pagnacco, F.Pierron Identification from measurements of mechanical fields. Experimental Mechanics. Volume : 48. $\mathrm{N}^{\circ} 4$. Pages : 381 402. 2008. Springer.

[3] M. Grediac,E.Toussaint, F.Pierron L'identification des propriétés mécaniques de matériaux avec la méthode des champs virtuels, une alternative au recalage par éléments finis. Comptes rendus Mécanique. Volume : 330. N2․ Pages : 107-112. 2002. Elsevier.

[4] W. Nunes dos Santos, P. Mummeryb , A. Wallwork: Thermal diffusivity of polymers by laser flash technique, Polymer Testing 24 (2005) 628-634.

[5] A. Germaneau and JC Dupré: Termam exchanges and termomechanical couplings in amorphous polymers, Polymers \& Polymer Composites, Vol. 16, No. 1, pp. 917, 2008

[6] K. Atchonouglo, M. Banna, C. Vallée and J.C Dupré, Inverse Transient Heat Conduction Problems and Application to the Estimation of Heat Transfer Coefficients, Heat and Mass Transfer, Vol. 45,Number 1, pp. 23-29, November 2008.

[7] K. Atchonouglo, Identification des Paramètre Caractéristiques d'un Phénomène Mécanique ou Thermique Régi par une équation différentielle ou aux dérivées Partielles, Thèse de Doctorat, Université de Poitiers, 2007.

[8] E. Barkanov, Introduction to the Finite Element Method, Institute of Materials and Structures, Faculty of Civil Engineering, Riga Technical University.

[9] P. Corde et A. Fouilloux, Cours Langage Fortran, 2008 